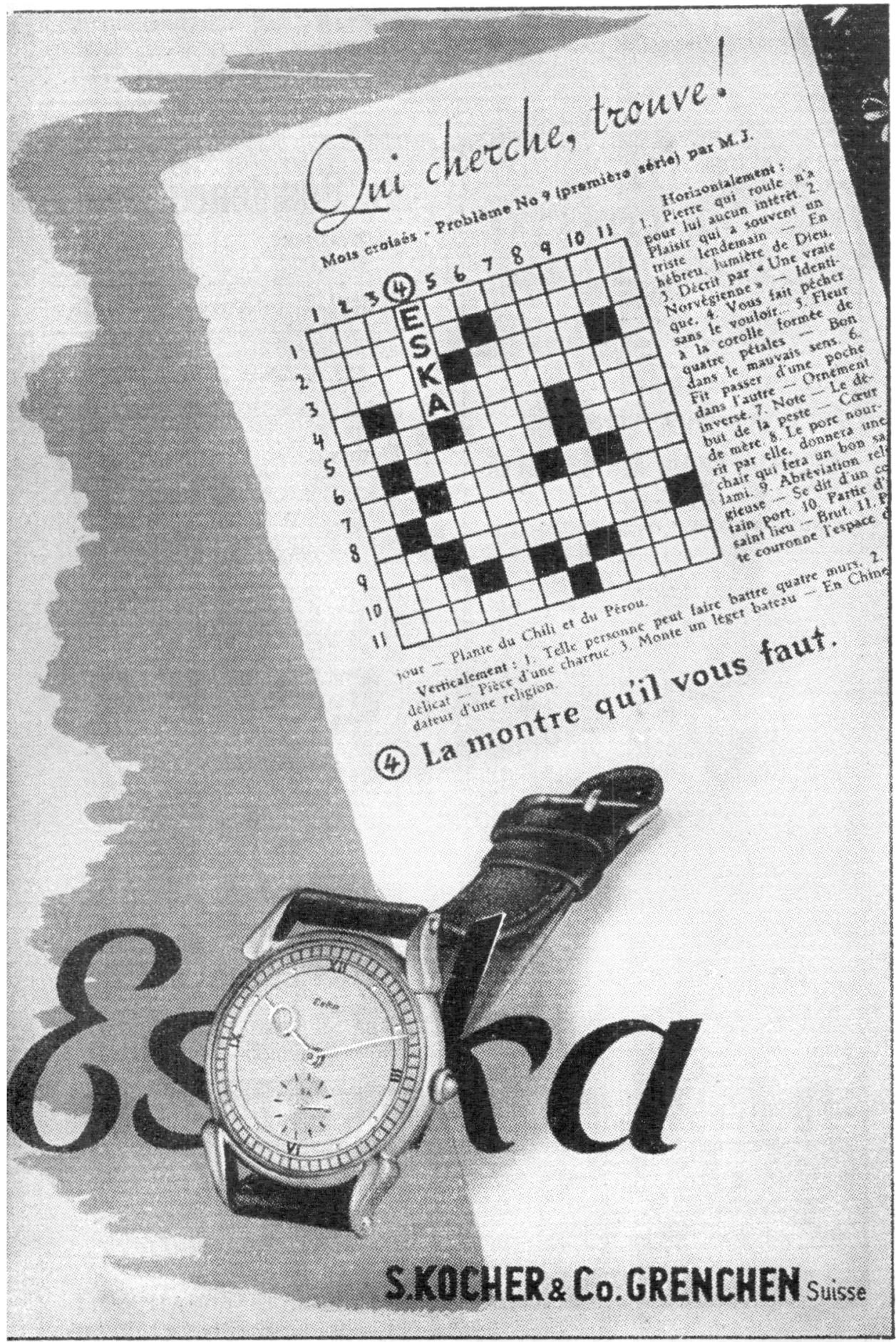




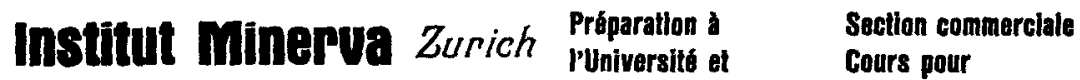 \\ l'Ecole Polytechnique aldes-médecins}

\section{UNIVERSITÉ \\ DE LAUSANNE}

Facultés de Théologie, Droit,

Médecine, Lettres et Sciences.

Ecoles de sciences sociales et politiques, hautes études commerciales, pharmacie.

Institut de police scientifique.

\section{ÉCOLE POLYTECHNIQUE ÉCOLE OE FRANÇAS MODERNE}

RENSEIGNEMEN IS

AU SECRETARIAT DE LUNIVERSITE

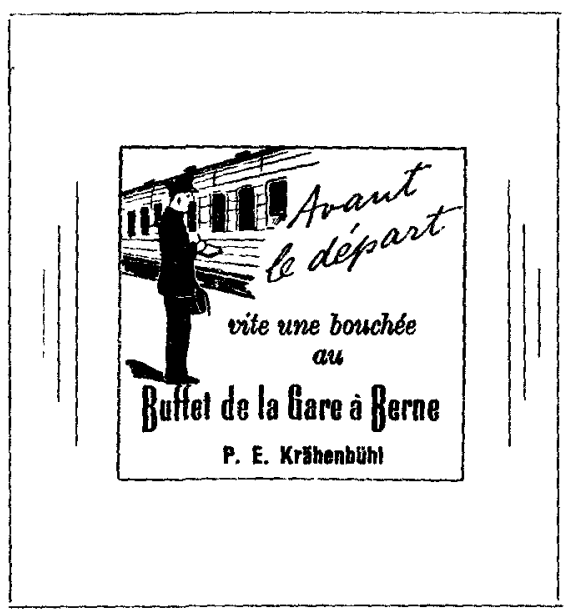

\section{La Résidence, Genève}

11, Florissant

Tél. 4 I 388

\section{Hôtel-Restaurant - Bar}

150 chambres avec téléphone

60 salles de bains

Tennis - Parc pour autos

Arrangements

pour familles

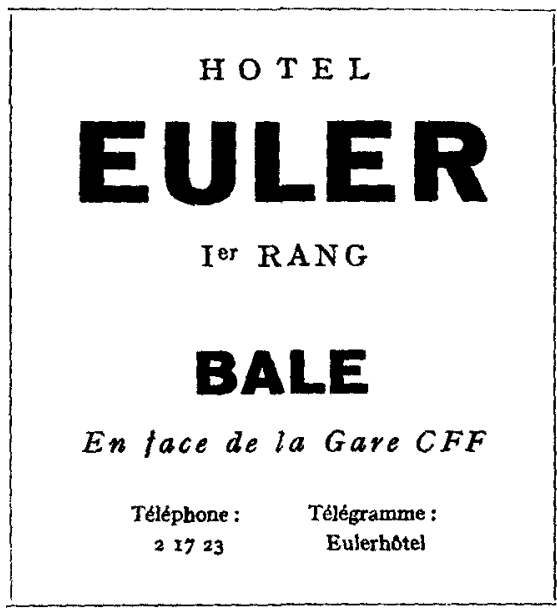

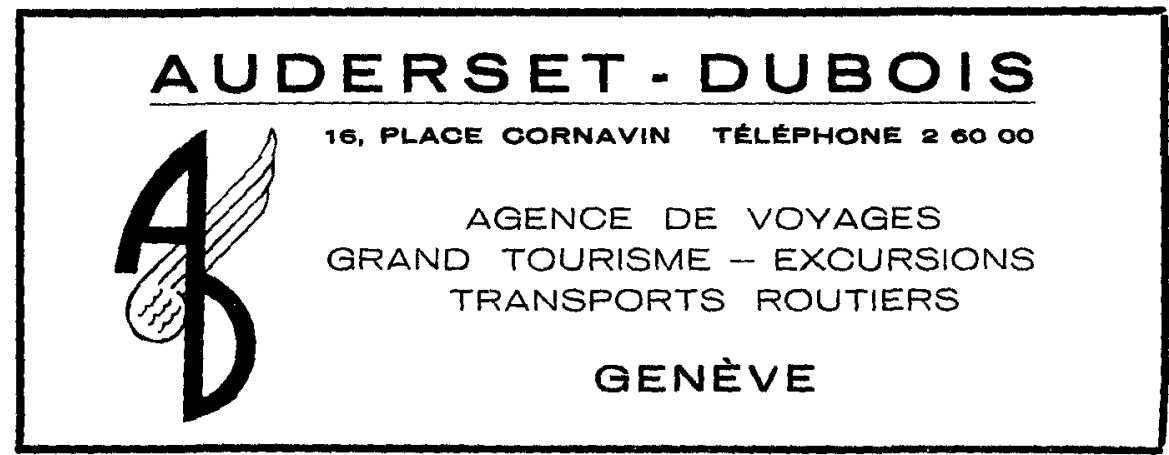




\title{
COMITE INTERNATIONAL DE LA CROIX-ROUGE
}

\author{
Membres du Conseil de la Présidence: \\ FRŔSIDENT : \\ PAUL RUEGGER \\ VICE•PRÉSIDENTS :

$\begin{array}{cc}\text { JACQUES CHENEVIERE } & \text { MARTIN BODMER } \\ \text { ERNEST GLOOR } & \text { LEOPOLD BOISSIER } \\ \text { MFMBRF: } & \\ \text { RENE VAN BERCHEM } & \text { RODOLFO OLGIATI }\end{array}$

Direction:

ROGER GALLOPIN

Directeur exécutif

DAVID DE TRAZ

Directeur exécutif-adjoint
JEAN S. PICTET

Directeur des Affaires générales

EDOUARD DE BONDELI

Sous-directeur

Secrétariat général:

JEAN DUCHOSAL

Secrétaire général 


\section{ADRESSES DES COMITES CENTRAUX DES SOCIETÉS NATIONALES DE LA CROIX-ROUGE}

ALBANIE - Croix-Rouge albanaise, Tirana.

ALLEMAGNE - Croix-Rouge allemande (Ouest). 67, Rheinweg, Bonn.

ARGENTINE - Croix-Rouge argentine, Hipólito Irigoyen 2068, Buenos-Aires.

AUSTRALIE - Croix-Rouge australienne, 122-128, Flinders Street, Melbourne.

AUTRICHE - Croix-Rouge a'itrichienne, Milchgasse 1, V'ienne I

BELGIQUE -- Croix-Rouge de Belgique, 98, Chaussée de Vleurgat, Bruxelles.

BIRMANIE - Croix-Rouge de Birmanie, Office of the direct or of public Health, Phayre Street, Rangoon.

BOLIVIE - Croix-Rouge bolivienne, Casilla num. 741, Lu Paz.

BRESIL - Croix-Rouge brésilienne, Praça de Criz Vermelha, 10-12, Rio de Janeiro.

BULGARIE - Croix-Rouge bulgare, 21, boul. Totleben, Sofia .

CANADA - Croix-Rouge canadienne, 95, Wellesley Street, Toronto, 5.

CHILI - Croix-Rouge chilienne, Calle Catedral, 1572, Correo, 15, Casilla, 246. V., Santiago de Chile.

CHINE - Croix-Rouge chinoise, 22 Kanmein Hutung, Pékin E.

COLOMBIE - Croix-Rouge colomibienne, Calle 8a, num. 7-73. Apartado, nacional 1110, Bogota.

COSTA-RICA - Croix-Rouge costaricienne, Calle 5a Sur, San José.

CUBA - Croix-Rouge cubaine, Zulueta, 461. La Havane.

DANEMARK - Croix-Rouge danoise, Platanvej 22 , Copenhague $V$.

REPUBLIQUE DOMINICAINE - Croix-Rouge domtnicaine Calle Galvan, 24, Apartado 1288, Ciudad Trujillo.

ÉGYPTE - Société nationale Fouad $I^{\text {er }}$ du Croissant-Rouge égyptien, rue Malika Nazli, 34, Le Caire.

ÉQUATEUR - Croix-Rouge équatorienne. Avenida Colombia y Elizalde, 118, Quito.

ISPAGNE - Croix-Rouge espagnole, Eduardo Dato, 18, Madrid.

ETATS-UNIS - Croix-Rouge américaine. National Headquarters, 17th and D. Streets, N.W., Washington 13, D.C

ÉTHIOPIE - Croix-Rouge éthiopienne, Addis-Abéba.

FINLANDE - Croix-Rouge de Finlande, Tehtaankatu I A, Helsinki.

FRANCE -- Croix-Rouge française, 17, rue Quentin-Bauchart, Paris $\left(8^{\mathrm{me}}\right)$.

GRANDE-BRETAGNE - Croix-Rouge britannique, 14, Grosvenor Crescent, Lonares S.W.1.

GRECE - Croix-Rouge hellénique, rue Mackenzie-King, 1 et rue Solonos, 39, Athines.

GUATÉMALA - Croix-Rouge du Guatémala, 4a, CalleOriente, 64, Guatémala.

HAIITJ - Croix-Rouge haîtienne, rue Férou, Port-au-Prince. HONDURAS - Croix-Rouge du Honduras, Tegucigalpa. HONGRIE - Croix-Rouge hongroise, Baross utca, 15, Budapest V'III.

INDE - Croix-Rouge de l'Inde, no 20, Talkatora Road, Nez? Delhi, 3.

INDONESIE - Croix-Rouge indonésienne, Djalan Sekolah, 8, Diakarta.

IRAK - Croissant-Rouge de l'Irak, Bagdad.

IRAN - Société du Lion et Soleil Rouges de l'Iran, Avenue Argue, Tehéran.
IRLANDE-Croix-Rouge irlandaise, 25 Westland Row, Dublin.

ISLANDE - Croix-Rouge islandaise, Thorvaidsensstraeti, 6 , Reykjavik.

ITALIE - Croix-Rouge italienne, 12, via Toscana, Rome.

JAPON - Croix-Rouge japonaise, 5, Shiba Park, Tokio.

JORDANIE - Croissant-Rouge jordanien. $A$ mman.

LIBAN - Croix-Rouge libanaise, Beyrouth.

LIECHTENSTEIN - Croix-Rouge du Liechtenstein, Vaduz.

LUXEMBOURG - Croix-Rouge luxembourgeoise, 5, rue de Rollingergrund, Luxembourg.

MEXIQUE - Croix-Rouge mexicaine, Esq. Durango y Monterrey Col. Roma, Mexico DF.

MONACO - Croix-Rouge monégasque, Monaco.

NICAR.AGUA-Croix-Rouge du Nicaragua, Managua D.N.C.A.

NORVEGE - Croix-Rouge de Norvège, Bankplassen, 1, Oslo.

NOUVELLE-ZELLANDE - Croix-Rouge néo-zélandaise, 61, Dixon Strcet, Welington C.1.

PAKISTAN - Croix-Rouge du Pakistan, Katrak Building, Mansfield Rodd, Karachi III.

PANAMA - Croix-Rouge de Panama, Panama.

PARAGUAY - Croix-Rouge paraguayenne, Avenida Espana, 505, A suncion.

PAYS-BAS - Croix-Rouge néerlandaise, 27, Princessegracht, La Have.

PEROU - Croix-Rouge péruvienne, Avenida Nicolas de Piérola, 224, Lima.

PHILIPPINES - Croix-Rouge philippine, 166, San Raphael Street, Manille.

POLOGNE - Croix-Rouge polonaise, Mokotowska, 14, Varsovie.

PORTUGAL - Croix-Rouge portugaise, Secrétariat général Praça do Comércio, 1, Lisbonne.

ROUMANIE - Croix-Rouge roumaine, Strada Biserica Amzei, 29, Bucarest.

SAINT-MARIN - Croix-Rouge de Saint-Marin, Saint-Marin.

SALVADOR - Croix-Rouge du Salvador, 3a, Calle Pomente, 21, San Suivador.

SARRE - Croix-Rouge sarroise, Gerberschule, Saarbrucken, 3 .

SIAM - Croix-Rouge siamoise, King Chulalongkom Memorial Hospital, Bangkok.

SUĖDE - Croix-Rouge suédoise, Artillerigatan, 6, Stockholm 14. SUISSE - Croix-Rouge suisse, Taubenstrasse, 8, Berne.

SYRIE - Croissant-Rouge syrien, Dasnas.

TCHÉCOSLOVAQUIE -- Croix-Rouge tchécoslovaque Thunovska, 18, Prague III.

TURQUIE - Société du Croissant-Rouge turc, Yenisehir, Ankara.

UNION SUD-AFRICAINE - Croix-Rouge sud-africaine, Red Cross House, 77 de Villiers Street, Johannesburg.

U.R.S.S. - Alliance des Sociétés de la Croix-Rouge et du Croissant-Rouge de l'U.R.S.S., rue Neglinnaya, 25, Moscou.

URUGUAY - Croix-Rouge uruguayentne, Avenida, 8 de Octubre, 2990, Montevideo.

VENEZUELA - Croix-Rouge vénézuélienne, Avenida Este, 303-3, Caracas.

YOUGOSLAVIE - Croix-Rouge yougoslave, Simına ulica broj, 19, Belgrade. 